\title{
Laser-induced non-linear light scattering in a suspension of black-body particles
}

\author{
S.E. Zelensky \\ Optics Division, Physics Department, Kyiv National Taras Shevchenko University, \\ 6, prospect Glushkova, 03680 Kyiv, Ukraine, E-mail: zele@univ.kiev.ua, phone (044)266-2295
}

\begin{abstract}
Characteristics of non-linear scattering of powerful pulses of $Q$-switched YAG: $\mathrm{Nd}^{3+}$ laser in an aqueous suspension of submicron-sized black-body particles has been investigated. Proposed is a model describing the results of experiments. This model involves laserinduced overheating of suspended particles and vaporization of surrounding water with subsequent rapid growth of vapor shells around the particles.
\end{abstract}

Keywords: laser-induced scattering, non-linear processes, black-body suspensions.

Paper received 03.03.04; accepted for publication 17.06.04.

\section{Introduction}

Interaction of powerful pulsed laser radiation with blackbody colloidal particles initiates a number of various nonlinear optical phenomena caused by laser-induced heating of particles. Primitive numerical estimations argue that pulsed laser radiation of moderate surface power density $\left(5 \ldots 50 \mathrm{MW} \mathrm{cm}{ }^{-2}\right)$ is capable to overheat a submicron-sized black-body particle to a temperature of thousands of Kelvins. The laser-induced incandescence (LII) of micro-particles is observed experimentally both in condensed matter and in gaseous phase [1-9]. The behavior of LII is well described using the particle energy balance equation together with Planck's formula for black-body thermal emission.

The laser-induced incandescence is employed for monitoring of soot particulate in flames and engine exhausts [1-5]. When a powerful pulsed laser irradiates microscopic black particles in a condensed matrix, acoustic waves are generated, which can be applied for detection of colloidal inclusions in ultra-pure liquids [10]. In transparent luminescent condensed matter containing suspended particles, LII can serve as a source of photoexcitation of luminescence, which enables to measure the luminescence quantum efficiency without the use of reference luminescent samples [8]. Non-linear reflection of pulsed laser radiation at a dielectric-carbon suspension interface can be exploited to produce optical switching in nanosecond timescale [11,12].
When a suspension of black-body particles is irradiated with a powerful pulsed laser, the phenomenon of optical power limiting is observed, which consists in nonlinear dependence of the cell transmittance on the incident laser fluence. With the increase of input laser power the cell transmittance decreases significantly, hence the output becomes clamped, which is useful for protection of optical devices against laser damage. Broadband optical limiting (from the visible to the infrared region) is observed in aqueous and other suspensions of black-body particles (for example, carbon black suspensions) $[13,14]$, carbon nanotubes [15-17], fullerene solutions [14], etc.

The primary cause of optical limiting in suspensions of absorbing particles is self-induced scattering of pulsed laser radiation. There are two basic mechanisms of laser-induced scattering. (i) The laser-induced heating of suspended particles is followed by the overheating and quick vaporization of the surrounding liquid; hence vapor bubbles (vapor shells) grow around the particles and the light scattering increases significantly. (ii) Thermionic emission from overheated particles leads to avalanche ionization in the laser field, and the resulting microplasma expands and strongly scatters laser light.

Concerning the relative contribution of the mentioned two mechanisms of non-linear laser-induced light scattering, the authors $[13,14]$ have drawn a conclusion that laser-induced formation of vapor bubbles becomes actual at relatively high intensities of laser radiation. On 


\section{S.E. Zelensky: Laser-induced non-linear light scattering in a suspension of ...}

the other hand, the authors [18,19] have performed an extensive time-resolved investigation of degenerate fourwave mixing in an absorbing colloidal suspension and concluded that the main mechanism of optical non-linearity at the laser surface power density of approximately $1.5 \mathrm{MW} \mathrm{cm}^{-2}$ is the rapid growth of vapor bubbles at the surfaces of the laser-heated particles. In [9], on the basis of the vapor-bubble mechanism of optical limiting, the authors have proposed a simple model for calculation of the parameters measured experimentally and obtained good agreement of the results of experiments and calculations.

In this paper, we examine the non-linear properties of laser-induced light scattering in an aqueous suspension of submicron-sized black-body particles.

\section{Experimental details}

The measurements were performed with an aqueous suspension of absorbing particles, which was prepared by diluting and filtering black gouache paint. In contradistinction to dye solutions, paints are suspensions of insoluble pigment particles in transparent liquids. Pigments of black paints are typically made of carbon or some other chemicals. The chemical composition of the pigment used in this work is unknown. However, chemical composition of the pigment particles seems to be a secondary factor for the experiments on laser-induced scattering. For example, experiments show, that LII and laser-induced scattering (optical limiting) are observed with various paints and even with turbid natural water.

Concerning the size of particles, typical paints contain particles of various shapes and sizes within the range of $0.01 \ldots 5 \mu \mathrm{m}$. For the suspensions investigated in this paper, nephelometric measurements have indicated the average particle radius of approximately $0.1 \mu \mathrm{m}$.

In this paper, optical measurements were performed with the use of a computer-controlled spectrometer with Q-switched YAG: $\mathrm{Nd}^{3+}$ laser (wavelength $1064 \mathrm{~nm}$, pulse duration 25 ns FWHM, repetition rate approximately $1 \mathrm{~s}^{-1}$ ). The laser was equipped with a diaphragm inside the resonator, which provided a smooth bell-shaped crossbeam distribution of laser power. The photodetectors operated in the integrating mode, i.e. they detected the energy of each single optical pulse. The averaging procedure was implemented in the software.

All the measurements were performed at room temperature. The investigated suspension was pumped through the optical cell with a flow rate of approximately $0.51 \mathrm{~min}^{-1}$, so that each laser pulse interacted with a "fresh" portion of the suspension.

\section{Results and discussion}

Consider the interaction of a powerful laser pulse of nanosecond duration with an aqueous suspension of blackbody particles. For the calculation of power of scattered laser light, $P_{S}$, as a function of incident laser surface power density, $F_{0}$, the model [9] can be used. This model implies the following processes. Within the laser pulse duration, microscopic particles are heated by laser radiation and transfer a portion of the obtained energy to the vaporization of surrounding water. The process can be conceived as a nucleation and growth of vapor shells around the particles. Denote $G$ the ratio of the particle energy transferred to the vaporization of surrounding water to the particle energy obtained from the laser beam, namely $G=\Lambda d m\left(F \sigma_{0} d t\right)^{-1}$, where $\Lambda$ is the latent heat of vaporization of water, $\Lambda d m$ is the mass of water vaporized during the time interval $d t, \sigma_{0}$ is the particle absorption cross-section at the laser wavelength, $F$ is the laser surface power density. As a first approximation, the parameter $G$ can be treated as a constant. According to [9], the value of $G$ is estimated approximately as $0.01-0.5 \%$ for aqueous suspensions of submicron particles. The model implies that the vapor shells grow during the laser pulse with the speed largely dominated by inertia-controlled processes. The radius of the core-shell particle can be estimated with the use of the particle energy balance equation together with the equation of ideal gas state. So, the volume of water vapor, $V$, increases with time in direct proportion to the integral of laser power density,

$V(t) \sim \int_{-\infty}^{t} F\left(f^{\prime}\right) d t^{\prime}$. For particles with the radius of $r_{0} \approx$ $\approx 0.1 \mu \mathrm{m}$, calculations show that the radius, $r$, of a coreshell particle at the end of the laser pulse can be approximately twice as large as $r_{0}$. The model also uses the wellknown classical relations between the particle scattering cross section and the particle radius, namely, $\sigma \sim r^{6}$ for $r<\lambda$ and $\sigma \sim r^{2}$ for $r>\lambda$. The model is implemented in computer codes, which allows calculating all the parameters measured experimentally in [9].

For calculation of the scattered light power as a function of the laser power, we can use the following reasoning. Consider the energy balance equation for a laser pulse propagating through the suspension. In the case of linear attenuation of the laser beam, the following equation can be written

$F_{0} S=T_{0} F_{0} S+P_{A}^{0}+P_{S}^{0}$

where $F_{0}$ is the surface power density of the incident laser beam, $S$ is the laser beam cross area, $T_{0}$ is the optical transmittance of the cell at the laser wavelength at low levels of the laser power density, $P_{A}^{0}$ and $P_{S}^{0}$ are, respectively, the absorbed and scattered power of the laser beam propagating through the cell. As far as the primary mechanism of the self-induced attenuation of the laser beam is the laser-induced scattering, to a first approximation we suppose that an increase of laser power does not cause the increase of absorption in the suspension but causes only the increase of scattering. Then, in the case of non-linear attenuation of the laser beam in the suspension, the energy balance can be written

$$
F_{0} S=T_{0} F S+P_{A}^{0}+P_{S}^{0}
$$




\section{S.E. Zelensky: Laser-induced non-linear light scattering in a suspension of ...}

where $T$ is the optical cell transmittance which depends on the laser power, $P_{S}$ is the scattered light power that is a non-linear function of the incident laser power $F_{0}$.

Now we introduce the following parameter

$$
\alpha=P_{A}^{0}\left(P_{A}^{0}+P_{S}^{0}\right)^{-1},
$$

which is a measure of the absorption processes as compared with the total attenuation of the laser beam propagating through the suspension in the linear regime of interaction. As far as the number density of particles in the suspension remains constant, the introduced parameter $\alpha$ is equal to the averaged ratio of absorption and extinction cross-sections of the particles. For example, for a spherical carbon particle with the radius of $0.1 \mu \mathrm{m}$, the absorption and extinction cross-sections at a wavelength of $1.06 \mu \mathrm{m}$ are respectively estimated as $3.68 \cdot 10^{-14} \mathrm{~m}^{2}$ and $0.833 \cdot 10^{-14} \mathrm{~m}^{2}[18]$, hence we can estimate $\alpha \sim 0.8$. By the order of magnitude, such an estimate is in agreement with the results of direct measurements of scattered laser power in carbon black suspensions [14].

With taking into account (3), equations (1) and (2) result in the following expression for the scattered power as a function of the incident laser power

$$
P_{S}=F_{0} S\left[1-T\left(F_{0}\right)-a\left(1-T_{0}\right)\right]
$$

Expression (4) enables us to calculate the dependence $P_{S}\left(F_{0}\right)$ for a given dependence $T\left(F_{0}\right)$. The characteristics $P_{S}\left(F_{0}\right)$ calculated for various $\alpha$ are given in Fig. $1, b$. The linear transmittance of $2 \mathrm{~cm}$-thickness cell is set to $T_{0}=0.73$. The calculations were performed with the use of the dependence $T\left(F_{0}\right)$ (plotted in Fig. 1,a), which is the best fit of the experimental data [9]. Circle points in Fig. 1,b,c are the results of measurements of the scattered light intensity at the wavelength of $1.06 \mu \mathrm{m}$ and pulse duration of $25 \mathrm{~ns}$. The measurements were performed in the same conditions as in [9]. The scattered light was detected with a PMT tube (FEU-83) at an angle of scattering of approximately $\pi / 2$. As seen from Fig. $1, b$, the dependence of $P_{S}\left(F_{0}\right)$ is essentially non-linear. At relatively small levels of laser power density, $F_{0}<3 \mathrm{MW} \mathrm{cm}^{-2}$, the slope of the curve $P_{S}\left(F_{0}\right)$ equals to unity (in a log-log scale). For the visualization, the dashed lines in Fig. 1, $b, c$ are drawn with a unit slope. With the increase of $F_{0}$ the scattering becomes non-linear and the slope of the curve increases up to approximately 2 . Further increase of $F_{0}$ leads to the decrease of the slope to nearly a unit.

As seen from Fig. 1, $b$, the experimental and theoretical data disagree. This disagreement can be explained by taking into account the following circumstance. The theoretical curves $P_{S}\left(F_{0}\right)$ given in Fig. 1, $b$ correspond to the integral scattered laser power over the whole volume and over all possible scattering angles, whereas the experiments were carried out at a fixed scattering angle. The considered mechanism of non-linear scattering implies that the scattering indicatrix depends on the laser power density and can change significantly during the laser pulse due to the increase of vapor shells.

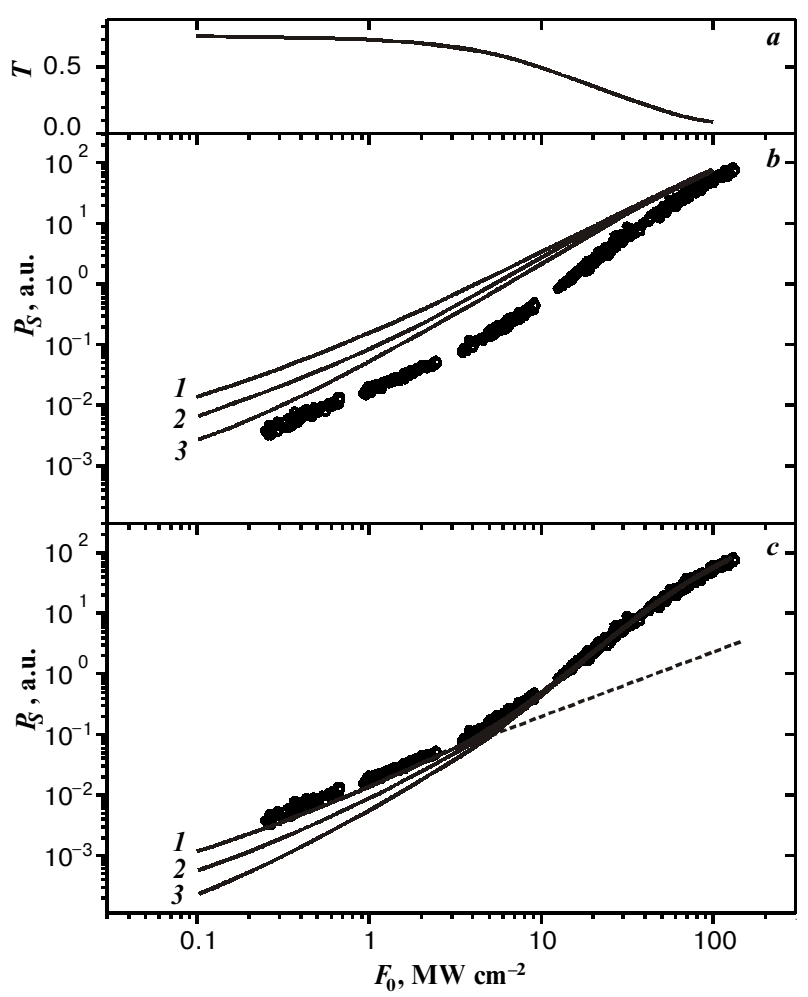

Fig. 1. The optical transmittance $(a)$ and scattered laser power $(b, c)$ as a function of the incident laser surface power density. Circle points - experiment, solid lines - calculations. Dashed lines indicate the unit slope. $b$-calculated according to equation (4), $c$ - calculated according to equation (8) from $z_{1}=0$ to $z_{2}=$ $=0.05 d$ for a cell thickness of $d=2 \mathrm{~cm} ; \alpha=0.5$ (1), 0.75 (2), 0.9 (3).

Following the methodology developed in previous papers $[6,7,20]$, for subsequent analysis, we introduce the factor of non-linearity for the scattered radiation as follows

$\gamma_{S}=\frac{d P_{S} / P_{S}}{d F_{0} / F_{0}}$.

The parameter (5) is a convenient dimensionless measure of non-linearity of the investigated process, which can be easily determined from the experimental data regardless of the units of measuring of $P_{S}$ and $F_{0}$. With the use of (4), by a little algebra, the following expression can be derived

$\gamma_{S}=1-\frac{\gamma_{T} T}{1-\alpha\left(1-T_{0}\right)-T}$,

where $\gamma_{T}$ is the following factor of non-linearity for the optical transmittance

$\gamma_{T}=\frac{d T / T}{d F_{0} / F_{0}}$,

which can be calculated from the known dependence $T\left(F_{0}\right)$. Equation (6) enables to calculate the dependence 


\section{S.E. Zelensky: Laser-induced non-linear light scattering in a suspension of ...}

of $\gamma_{S}\left(F_{0}\right)$ with the use of $T\left(F_{0}\right)$ from Fig. 1,a. Fig. 2,b shows the results of calculations for various values of $\alpha$. It is worth noting that the obtained dependence of $\gamma_{S}\left(F_{0}\right)$ is a bell-shaped function, i.e. the investigated process is a representative of the processes with variable factors of non-linearity [20].

The experimental data for $\gamma_{S}\left(F_{0}\right)$ are given in Fig. 2,a. These data were collected at the same conditions as the data given in Fig. 1. As seen from Fig. 2,a, the experimental dependence of $\gamma_{S}\left(F_{0}\right)$ shows a clear maximum; this fact is in qualitative agreement with the calculations. However, it should be noted that the position of the maximum in Fig. 2, $a$ and its height differ from the calculated values (Fig. 2,b). The observed disagreement can not be explained only by the errors of measurements of the laser surface power density. Thus, the situation requires additional analysis.

As it was already mentioned, the calculations account the integral power of scattered laser light, whereas the measurements were carried out so that the photodetector collected the scattered light only from a small portion of the laser-irradiated volume. The range of vision of the photodetector covered only 5 to $10 \%$ of the length, $d$, of the laser-irradiated track near the entrance window of the optical cell. According to this, a computer program was developed that calculates the power of laser light

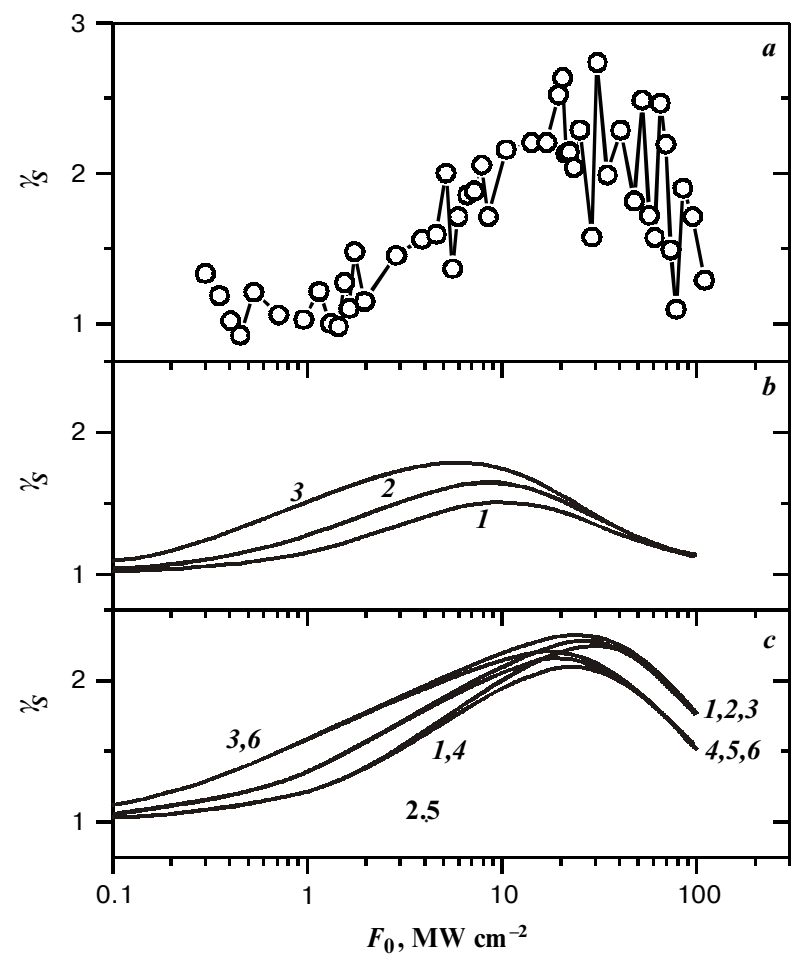

Fig. 2. The factor of non-linearity for scattered laser power as a function of the incident laser surface power density. Circle points $a$ - experiment, solid lines $b, c$-calculations; $b$-calculated according to equation (6); $c$ - calculated according to equation (8) from $z_{1}=0$ to $z_{2}=0.05 d(1-3)$ and to $z_{2}=0.01 d(4-6) ; \alpha=0.5$ $(1,4), 0.75(2,5), 0.9(3,6)$. scattered from a given region of the laser-irradiated track. Denote $z$ the coordinate along the laser beam axis. Then, for a given range from $z_{1}$ to $z_{2}$, the scattered laser power can be calculated as follows

$$
P_{S}=\int_{z_{1}}^{z_{2}} d z \int d t \sigma(z, t) F(z, t) N,
$$

where $N$ is the number density of particles in the suspension, $\sigma$ is the scattering cross-section of a core-shell particle, it depends on the irradiation history of the particle and therefore depends on $z$ and $t$. For calculation of $\sigma(z, t)$ and $F(z, t)$, the model [9] is employed. The integration over $t$ in (8) is performed within the extent of the laser pulse, which is approximated by a Gaussian function. The dependence of $P_{S}\left(F_{0}\right)$, calculated according to (8), was used for calculation of the factor of non-linearity, $\gamma_{S}$, according to its definition (6), as a function of the incident laser power density.

The theoretical curves $\gamma_{S}\left(F_{0}\right)$ calculated for the track regions adjacent to the entrance window of the cell are given in Fig. 2,c. By comparing Fig. 2, $a$ and Fig. 2,c, one can see that the theoretical curve $\gamma_{S}$ calculated for the range from $z_{1}=0$ to $z_{2}=0.05 d$ and for $\alpha=0.5$ is in good agreement with the experimental data.

The proposed improved method of calculation of scattered optical signals (8) enables to make a better fit of the experimental data presented in Fig. 1. The theoretical curves $P_{S}\left(F_{0}\right)$, calculated for the range from $z_{1}=0$ to $z_{2}=$ $=0.05 d$, are given in Fig. $1, c$ as solid lines. As seen, good agreement between the calculated and experimental data is obtained for $\alpha=0.5$.

\section{Concluding remarks}

In this paper, we investigated some peculiarities of the laser-induced light scattering in aqueous suspensions of absorbing particles. The model of interaction of powerful nanosecond laser pulses with the suspensions is improved. It is assumed that the primary mechanism of laser-induced scattering is the rapid growth of vapor shells around the suspended particles during the action of laser pulses. Despite its simplicity, the model enables to calculate all the experimentally measured characteristics of the investigated object, with good agreement between the experimental and calculated data.

\section{References}

1. Wal R.L. Vander, Laser-induced incandescence: detection issues // Appl. Opt., 35(33), pp. 6548-6559 (1996).

2. Wal R.L. Vander and K.J. Weiland, Laser-induced incandescence: Development and characterization towards a measurement of soot-volume fraction // Appl.Phys.B, 59, pp. 445-452 (1994).

3. B. Axelsson, R. Collin and P.E. Bengtsson, Laser-induced incandescence for soot particle size and volume fraction measurements using on-line extinction calibration // Appl. Phys. B, 72(3), pp.367-372 (2001). 


\section{S.E. Zelensky: Laser-induced non-linear light scattering in a suspension of ...}

4. F. Cignoli, S. Benecchi and G. Zizak, Time-delayed detection of laser-induced incandescence for the two-dimensional visualization of soot in flames // Appl. Opt., 33(24), pp. 57785782 (1994).

5. C. Allouis, F. Rosano, F. Beretta and A. D'Alessio, A possible radiative model for micronic carbonaceous particle sizing based on time-resolved laser-induced incandescence // Meas. Sci. Technol., 13, pp. 401-410 (2002).

6. S. Zelensky, Laser-induced heat radiation in borate glass // J.Phys.: Condens.Matter, 10, pp. 7267-7272 (1998).

7. S. Zelensky, Laser-induced heat radiation of suspended particles: a method for temperature estimation // J. Opt. A: Pure Appl.Opt., 1, pp. 454-458 (1999).

8. S. Zelensky, Laser-induced incandescence of suspended particles as a source of excitation of dye luminescence // J. Lumin., 104, pp. 27-33 (2003).

9. S.E. Zelensky, Self-induced attenuation of pulsed laser radiation in an aqueous suspension of submicron light-absorbing particles // J.Phys.: Condens. Matter, 15, pp. 6647-6657 (2003).

10. T. Kitamori, K. Yokose, M. Sakagami and T. Sawada, Detection and counting of ultrafine particles in ultrapure water using laser breakdown acoustic method // Jpn. J. Appl. Phys., 28, pp. 1195-1198 (1989)

11. C.M. Lawson and R.R. Michael, Nonlinear reflection at a dielectric-carbon suspension interface: Macroscopic theory and experiment // Appl.Phys.Lett., 64(16), pp. 2081-2083 (1994).
12. C.M. Lawson, G.W. Euliss and R.R. Michael, Nanosecond laser-induced cavitation in carbon microparticle suspensions: Applications in nonlinear interface switching // Appl. Phys. Lett., 58(20), pp.2195-2197 (1991).

13. K. Mansour, M.J. Soileau and E.W. Van Stryland, Nonlinear optical properties of carbon-black suspensions (ink) // J. Opt. Soc. Am., B9(7), pp. 1100-1109 (1992).

14. K.M. Nashold, and D.P. Walter, Investigations of optical limiting mechanisms in carbon particle suspensions and fullerene solutions// J. Opt. Soc. Am., B12(7), pp. 1228-1237 (1995).

15. Z. Jin, L. Huang, S.H. Goh, G. Xu and W. Ji, Size-dependent optical limiting behavior of multi-walled carbon nanotubes I/ Chem. Phys. Lett., 352, pp. 328-33 (2002).

16. P. Chen, X. Wu, X. Sun, J. Lin, W. Ji and K.L. Tan, Electronic structure and optical limiting behavior of carbon nanotubes // Phys.Rev.Lett., 82(12), pp. 2548-2551 (1999).

17. X. Sun, Y. Xiong, P. Chen, J. Lin, W. Ji, J.H. Lim, S.S. Yang, D.J. Hagan and E.W. Van Stryland, Investigation of an optical limiting mechanism in multiwalled carbon nanotubes // Appl. Opt., 39(12), pp. 1998-2001 (2000).

18. K.J. McEwan and P.A. Madden, Transient grating effects in absorbing colloidal suspensions // J.Chem.Phys., 97(11), pp. 8748-8759 (1992).

19. H. Lowen and P.A. Madden, A microscopic mechanism for shock-wave generation in pulsed-laser-heated colloidal suspensions // J. Chem. Phys., 97(11), pp. 8760-8766 (1992).

20. S.E. Zelensky, Non-uniformity of cross-beam laser power distribution as a source of errors in non-linear spectroscopy // Semicond. Phys., Quant. Electron. and Optoelectron., 6(3), pp. 378-381 (2003). 